\title{
Les anticorps monoclonaux en neurologie
}

$>$ L'utilisation des anticorps thérapeutiques commence à se développer avec succès en neurologie. Leur efficacité est conditionnée par la capacité à contourner la principale limite à l'utilisation de ces molécules dans ce type d'indication qu'est l'accessibilité au cerveau. Le caractère multifactoriel de ces pathologies neurologiques rend également complexe l'identification d'une cible spécifique. Nous exposons dans cette revue les effets neuroprotecteurs des anticorps monoclonaux et résumons leurs activités sur les mécanismes neurodégénératifs et inflammatoires. Les anticorps monoclonaux, tels que le natalizumab, représentent une avancée importante dans le traitement de la sclérose en plaque (SEP) et sont désormais utilisés en routine. Ce type de thérapeutique est toujours en développement dans les maladies neurodégénératives, en agissant principalement sur l'agrégation des protéines mal repliées, telles que la protéine béta-amyloïde et la protéine tau. Une autre stratégie en développement consiste à bloquer les inhibiteurs physiologiques de croissance axonale et de myélinisation, tels que Nogo-A et son co-transporteur LINGO-1. L'autorisation récente par la food and drug administration américaine des anticorps monoclonaux anti-calcitonin gene-related peptide (CGRP) dans la migraine a soulevé un regain d'intérêt pour ces thérapeutiques en neurologie. La recherche est de ce fait très active pour améliorer les formes galéniques et les voies d'administration et pour étendre ces thérapeutiques à d'autres cibles. <

L'utilisation des anticorps monoclonaux (AcM) figure parmi les avancées les plus significatives de cette dernière décennie dans tous les domaines, y compris la neurologie. La neurologie n'a pas été pionnière, et a plutôt suivi le sillage des succès qui commençaient à se
Hélène Blasco ${ }^{1}$, Pierre-François Pradat ${ }^{2,3}$

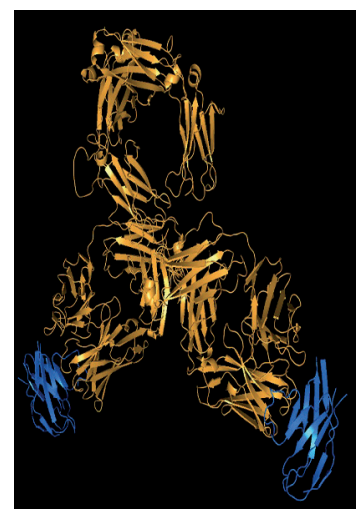

${ }^{1}$ Université de Tours, Inserm U1253, laboratoire de biochimie, CHRU de Tours, France

²Département des Maladies du Système Nerveux, Centre Référent Maladie Rare SLA, Hôpital de la Pitié-Salpétrière, Paris, France.

${ }^{3}$ Sorbonne Université, UPMC Univ Paris 6, CNRS, Inserm, laboratoire d'imagerie biomédicale, Paris, France.

helene.blasco@univ-tours.fr

faire marquants dans d'autres domaines de la médecine, notamment en cancérologie. Parmi les facteurs expliquant ce retard, on pourrait évoquer la relative complexité des maladies neurologiques et la méconnaissance de leurs mécanismes qui sont multiples et intriqués. Il était dès lors difficile de cibler un mécanisme causal ou au moins jouant un rôle critique dans la cascade biologique conduisant aux manifestations neurologiques. Les insuffisances des modèles animaux sont un autre facteur, la complexification du système nerveux au cours de la phylogénèse rendant hasardeuse la translation des résultats obtenus lors d'études précliniques aux essais thérapeutiques chez l'homme. Mais au-delà de considérations théoriques, un facteur limitant les traitements par AcM est l'existence de la barrière hématoencéphalique (BHE) qui rend difficile le passage de molécules de haute masse moléculaire.

Cet obstacle peut être toutefois contourné par divers moyens : simplement parce que la cible peut se situer en dehors du système nerveux, ou parce que la nature même du processus pathologique fragilise cette barrière. On peut aussi envisager un mode d'administration différent de la voie systémique, comme la voie intrathécale. Dans cette revue, nous nous attacherons à tracer les caractéristiques des processus pathogéniques apportant un rationnel pour une approche thérapeutique fondée sur les $A c M$ et, pour chacun d'eux, les avancées concrètes en thérapeutique. Cette revue ayant pour but d'être brève et synthétique, nous avons pris le parti d'omettre les pathologies tumorales ainsi que les affections du système nerveux périphérique.

\section{Juguler l'inflammation}

La sclérose en plaques (SEP) a été la première pathologie et, en fait, longtemps la seule, pour laquelle ont été utilisés des anticorps monoclonaux dans le domaine de la neurologie. Plusieurs facteurs 
expliquent cette primauté qui a permis des succès thérapeutiques majeurs. Tout d'abord, il s'agit d'une pathologie dont la physiopathologie est relativement bien connue et qui fait intervenir deux mécanismes généraux, l'inflammation et l'auto-immunité. Ces mécanismes retrouvés dans d'autres pathologies, notamment les rhumatismes inflammatoires chroniques, ont été largement étudiés et ont permis le développement de thérapeutiques innovantes pouvant ainsi être mis à profit dans la SEP. L'existence de biothérapies déjà efficaces contre l'inflammation dans la SEP, comme les interférons ou l'acétate de glatiramère, constituait également un contexte favorable. Un autre facteur essentiel est que la nature du processus pathologique responsable de la SEP permet de s'affranchir des limites d'accessibilité au cerveau par la barrière hémato-encéphalique. En effet, cibler les lymphocytes circulants, dont l'activation périphérique est le facteur initial de la réaction auto-immune dirigée contre les constituants de la myéline, est une stratégie pertinente. Le premier anticorps monoclonal approuvé dans la SEP a été le natalizumab qui se fixe à la sous-unité $\alpha 4$ de l'intégrine $\alpha 4 \beta 1$ (ou VLA-4 pour very late antigen-4) présente à la surface des lymphocytes $T$. L'intégrine $\alpha 4 \beta 1$ se fixe sur la molécule d'adhérence VCAM (vascular cell adhesion protein) exprimée par les cellules endothéliales de la BHE. Le natalizumab bloque le passage des lymphocytes T exprimant l'intégrine $\alpha 4$ à travers la $B H \varepsilon$, diminuant ainsi I'inflammation au niveau du SNC. Les patients traités ont montré une diminution de $70 \%$ de leur taux de poussées et une amélioration des lésions, par imagerie par résonnance magnétique (IRM), qui n'avait jamais été objectivée avec les traitements antérieurs [1]. La survenue bien que rare d'encéphalopathies multifocales progressives, pathologies opportunistes graves liées au virus JCV, limite toutefois son utilisation aux formes actives de SEP. Depuis, d'autres AcM, utilisés initialement pour le traitement d'hémopathies malignes, ont été développés par les laboratoires pharmaceutiques dans cette indication, comme l'alemtuzumab (qui cible la molécule CD52 sur les lymphocytes et monocytes), l'ocrelizumab et le rituximab (tous les deux ciblant l'antigène CD20 exprimé à la surface des lymphocytes B) [2]. Le tocilizumab, un AcM anti-IL(interleukine)-6, acteur central de l'inflammation, qui bloque sa fixation à son récepteur (IL6-R) et donc l'activité pro-inflammatoire de cette cytokine, a fait l'objet d'un essai de phase II.

\section{Capturer les protéines dépliées}

Une hypothèse unificatrice dans les maladies neurodégénératives fait jouer un rôle central à l'agrégation de protéines mal repliées : l'amyloïde beta (maladie d'Alzheimer), l'alpha-synucléine (maladie de Parkinson), la protéine tau (maladie d'Alzheimer, paralysie supranucléaire progressive), la TDP-43 (TAR DNA-binding protein 43) (majorité des scléroses latérales amyotrophiques [SLA], certaines démences temporo-frontales) ou encore la protéine SODl (superoxide dismutase 1) (certaines formes familiales de SLA). Cibler ces protéines toxiques reste actuellement une stratégie de choix dans ces pathologies et représente la principale justification à l'utilisation d'AcM.
L'hypothèse amyloïde dans la maladie d'Alzheimer ayant connu une situation de monopole pendant plus de 25 ans, la protéine amyloïde beta a été la cible choisie pour les premiers essais de neuroprotection par des AcM [3]. Ces essais, initiés avec beaucoup d'espoir et d'enthousiasme, ont certes permis une diminution de la charge en protéine amyloïde mesurée par tomographie par émission de positons (PET scan) mais n'ont apporté qu'une amélioration clinique minime, voire discutable. Ils ont de plus exposé les patients à des complications sous la forme d'amyloid-related imaging abnormalities (ARIA) pouvant entraîner des œdèmes cérébraux dus à des dépôts d'amyloïde bêta présents dans les vaisseaux cérébraux. En dehors des stratégies visant à diminuer les effets des ARIA (par exemple en améliorant le passage de la BHE afin de délivrer des doses moindres), une tendance actuelle forte est de sélectionner dans les essais thérapeutiques, des patients à un stade très précoce de la maladie afin d'optimiser les chances d'interrompre ou de limiter la cascade de l'agrégation de l'amyloïde beta. Trois anticorps sont actuellement évalués dans des essais de phase III : ganténérumab, aducanumab et crénézumab. Dans l'avenir, ce sera peut-être en intervenant à un stade pré-symptomatique, ce qui est envisageable grâce au développement de biomarqueurs, que cette approche sera plus susceptible d'être efficace. Une autre stratégie est de cibler la protéine tau hyperphosphorylée, dont l'accumulation est mieux corrélée avec la démence que la protéine amyloïde bêta [4]. Fondés sur la bonne tolérance des anticorps anti-tau, des essais de phase II sont actuellement en cours dans des formes précoces de maladie d'Alzheimer, mais également dans une autre taupathie, la paralysie supranucléaire progressive [5].

\section{Préserver les axones et la myéline}

Cette stratégie a été utilisée dans la SLA contre la protéine Nogo-A, un inhibiteur de croissance axonale, dont la surexpression est corrélée avec la progression de la maladie [6]. Toutefois, un essai de phase II utilisant un AcM anti-Nogo-A s'est révélé négatif [7]. La protéine Nogo-A, mais également son co-transporteur LINGO-1 (leucine-rich repeat and Ig domain-containing 1), font également l'objet de développements thérapeutiques dans la SEP. Le blocage de LINGO-1, qui est surexprimé à la surface des oligodendrocytes et des neurones dans la SEP, pourrait diminuer la destruction des gaines de myéline, préserver les axones, et aider à la remyélinisation [8-9]. 


\section{Limiter la vasodilatation cérébrale et la douleur}

L'avènement des AcM anti-CGRP (calcitonin gene-related peptide) (érénumab, frémanézumab, galcanézumab) est considéré comme le progrès le plus important jamais réalisé dans le domaine de la migraine. Le rationnel repose sur de nombreuses études qui ont montré que le CGRP avait un effet vasodilatateur et est impliqué dans la douleur. Ces traitements ont fait la preuve de leur efficacité dans des essais de phase III en montrant notamment une diminution de moitié de la durée des crises migraineuses chez la moitié des patients et une amélioration de la capacité à réaliser des activités quotidiennes. Ce traitement bénéficie de l'autorisation de la FDA depuis mai 2018.

\section{Conclusion}

À travers les différents exemples d'utilisation des AcM en neurologie que nous avons décrits, nous pouvons être confiants dans la poursuite de ces développements. L'utilisation de ces agents thérapeutiques très spécifiques de cibles moléculaires en neurologie, domaine dans lequel l'impasse thérapeutique est marquée, fait écho à la recherche très active et fructueuse sur les biomarqueurs dans cette discipline. Les avancées réalisées sur les agents thérapeutiques dérivés d'anticorps comme les nanobodies, qui permettent un accès plus facile au cerveau, les modes d'administration améliorant potentiellement la distribution dans le cerveau (voie intra-nasale par exemple), permettent d'envisager un avenir prometteur pour ce type de thérapeutiques. La recherche sur les mécanismes physiopathologiques de certaines maladies neurologiques nous amène de plus à envisager une action thérapeutique efficace en périphérie et pas uniquement au niveau central, élargissant ainsi le type d'anticorps utilisable. $\diamond$

\section{SUMMARY}

\section{Monoclonal antibodies in neurology}

Therapeutic antibodies have been successfully developed in neurology. However, their efficacy needs to overcome a main hurdle that is their limited access to the brain. Moreover, the multifactorial characteristics of many neurological diseases complicate the identification of a specific target. In this review, we present the neuroroprotective effect of some monoclonal antibodies and we summarize how they can interact with neurodegenerative and inflammatory processes. Monoclonal antibodies, such as natalizumab, that represent a major achievement in multiple sclerosis, have been approved in this indication and used in the routine practice. Also, they are still in a phase of development in degenerative diseases and mainly aim to counteract the aggregation of misfolded proteins such as amyloid beta or tau proteins. Another strategy in development of new monoclonal antibodies is to block physiological inhibitors of axonal growth and myelination such as Nogo-A or its co-transporter LINGO1. Finally, the recent approbation by the FDA of monoclonal antibodies against CGRP for migraine treatment has been a major breakthrough that expanded the potential fields of application of antibodies in neurology. Thus, a lot of research efforts are now devoted to improve the galenic forms, the routes of administration and to extend these various approaches to other targets. $\diamond$

\section{LIENS D'INTÉRÊT}

Les auteurs déclarent n'avoir aucun lien d'intérêt concernant les données publiées dans cet article.

\section{RÉFÉRENCES}

1. Polman $\mathrm{CH}, \mathrm{O}^{\prime}$ Connor PW, Havrdova $\varepsilon$, et al. A randomized, placebocontrolled trial of natalizumab for relapsing multiple sclerosis. $N$ Engl J Med $2006 ; 354: 899-910$.

2. Tintore $M$, Vidal-Jordana A, Sastre-Garriga J. Treatment of multiple sclerosis: success from bench to bedside. Nat Rev Neurol 2019 ; 15 : 53-8.

3. Schilling S, Rahfeld JU, Lues I, et al. Passive $A \beta$ immunotherapy: current achievements and future perspectives. Molecules 2018; 23.

4. Braak H, Braak $\varepsilon$. Neuropathological staging of Alzheimer-related changes. Acta Neuropathol $1991 ; 82$ : 239-59.

5. Gerson J, Kayed R. Therapeutic approaches targeting pathological tau aggregates. Curr Pharm Des 2016 ; 22 : 4028-39.

6. Pradat PF, Bruneteau G, Gonzalez de Aguilar JL, et al. Muscle Nogo-A expression is a prognostic marker in lower motor neuron syndromes. Ann Neurol $2007 ; 62: 15-20$.

7. Meininger V, Genge A, van den Berg LH, et al. NOG112264 study group. Safety and efficacy of ozanezumab in patients with amyotrophic lateral sclerosis: a randomised, double-blind, placebo-controlled, phase 2 trial. Lancet Neurol $2017 ; 16: 208-16$.

8. Foale S, Berry M, Logan A, et al. LINGO-1 and AMIG03, potential therapeutic targets for neurological and dysmyelinating disorders? Neural Regen Res $2017 ; 12: 1247-51$

9. Ineichen BV, Plattner PS, Good N, et al. Nogo-A antibodies for progressive multiple sclerosis. CNS Drugs 2017 ; 31 : 187-98.

\section{TIRÉS À PART}

H. Blasco

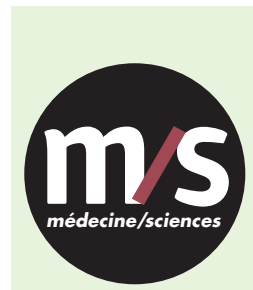

Tarifs d'abonnement $\mathrm{m} / \mathrm{s}-2019$

Abonnez-vous

à médecine/sciences
$>$ Grâce à $\mathrm{m} / \mathrm{s}$, vivez en direct les progrès des sciences biologiques et médicales

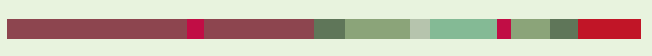

Bulletin d'abonnement page 1230 dans ce numéro de $\mathrm{m} / \mathrm{s}$
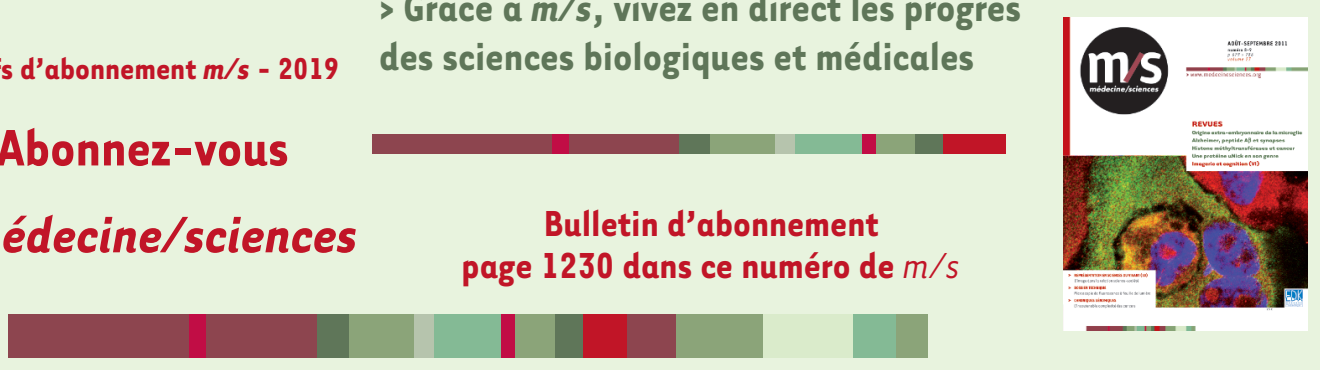